\title{
INVESTIGATION OF OBSTACLE EFFECTS ON THE AERODYNAMIC PERFORMANCE OF FLAPPING WINGS
}

\author{
Bo Yin* \\ Key Laboratory for Mechanics in \\ Fluid Solid Coupling Systems \\ Institute of Mechanics \\ Chinese Academy of Sciences \\ Beijing China \\ Email: yinbo@imech.ac.cn
}

\author{
Guowei Yang \\ Key Laboratory for Mechanics in \\ Fluid Solid Coupling Systems \\ Institute of mechanics \\ Chinese Academy of Sciences \\ Beijing China \\ Email: gwyang@imech.ac.cn
}

\begin{abstract}
The study of highly unsteady wing flapping includes the large scale vortices, complicated locomotion/dynamics and deformable wing structures. When flapping insects/birds approach or perch on some objects, such as ground, wall or obstacle, the solid boundary dissipates, absorbs and bounces the leading edge, trailing edge and wing tip vortices, which are generated and shed during the flapping flight. Such phenomenon creates a high pressure area, leads to cushion effect and influences the aerodynamics, stability and maneuverability significantly. This paper uses immersed boundary method (IBM) to numerically study the aerodynamic performance of flapping wing in proximity of obstacles, investigate the distance, flapping motion and wing flexibility effects and relevant symmetric/asymmetric flow patterns, research the influence of vortex generating and shedding to the lift/drag change, explore the key distance and reveal the mechanism how insects/birds adjust the flapping motion to achieve ideal flight. Such research could theoretically support the development of micro-bionic flapping wing vehicle.
\end{abstract}

\section{NOMENCLATURE}

A Flapping amplitude

$f$ Flapping frequency

$\alpha$ Rotation amplitude

$c$ Chord length of flapping wing

d Diameter of obstacle

$D$ Distance between wing and obstacle

*Address all correspondence to this author.

\section{INTRODUCTION}

Scientists have been studying the micro-aerial vehicle (MAV) for decades. Among these researches, aerodynamics of insect flight has drawn considerable attention in recent years due to its promising application in the development of biomimetic MAV [1].

Ideal biomimetic MAV with flapping wings is of small scale, high maneuverability, low energy consumption and noise, high lift and thrust generation, and high controllability. Insects adjust the flapping amplitude, frequency and mode to accommodate to various environments, which may be more efficient than fixed wings [2]. In nature, insects often approach or perch on some objects such as flowers, grass and rocks. Under such circumstances, the boundary effect influences aerodynamics and stability greatly $[3,4]$. To study the characteristics of flapping flight in finite space, especially interacting with ground, cliff or obstacle, will help us understand how the aerodynamic forces, flight mode and stability are affected and provide new perspective on MAV design. Researchers have done extensive work on the infinite ground effect numerically and experimentally. However, the knowledge of finite obstacle effect is very limited. Therefore the purpose of this paper is to investigate the obstacle effect on the hovering flight by constructing simplified computational model.

Because the quasi-steady theory fails to explain the unsteady, large angle of attack (AoA) and high lift flapping flight, Ellington [5] pointed out that the stall delay mechanism due to the leading-edge vortex (LEV) remarkably enhance the lift force. Wake capture and quick rotation also contribute to the lift increase and force peak at the end of flapping cycle [6,7]. Wang [8] and Shyy [9] numerically simulated the insect flight, analyzed the unsteady aerodynamics and validated the aforementioned mechanisms. Further, ground effect has been considered when study- 


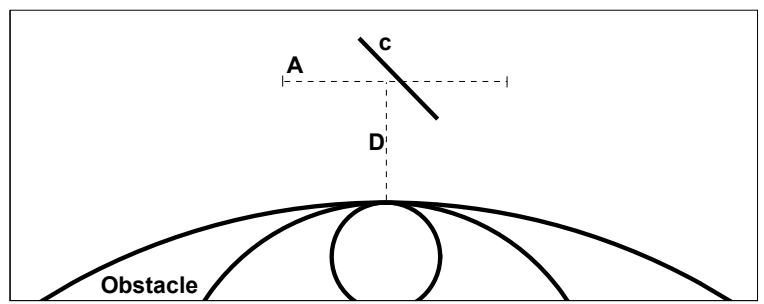

FIGURE 1. SCHEMATIC OF THE WING DURING NORMAL HOVERING FLIGHT IN PROXIMITY OF DIFFERENT SIZED OBSTACLES.

ing the unsteady flapping flight [10]. Three stages of aerodynamic force change have been observed depending on the distance to the groud: increase, decrease and recover. The interaction among the LEV, TEV and ground contributes to the force changes, which differs obviously with the free space flight. Besides, 2D simulation has also been extended to 3D research [11], which draws consistent conclusion with 2D study. Three dimensional tip vortex affects the forces significantly and the cushion effect under the wings plays key role in near ground flight. Recently, Wang and Yeung [12] studied the finite partial ground effect, which helps to understand the aerodynamics when the MAV lands on aerial platform. According to the reviews, our paper further investigate the finite obstacle effect on the aerodynamics when hovering motion gets close to the surface.

\section{METHODS}

\section{Problem Description And Numerical Approach}

We consider a two-dimensional hovering wing section with the chord length $c$, as shown in Fig. 1. The wing undergoes a combined translational and rotational motion specified at the leading edge [13],

$$
\begin{aligned}
x(t) & =\frac{A}{2} \cos (2 \pi f t) \\
\alpha(t) & =\alpha_{0}+\beta \sin (2 \pi f t+\phi)
\end{aligned}
$$

where $x(t)$ is the horizontal position of the leading edge, $\alpha(t)$ is the angle between the leading edge and the horizontal axis (measured in the counterclockwise direction), $A$ is the stroke distance of the leading edge - flapping amplitude, $\alpha_{0}$ is the initial orientation, $\beta$ is the angle amplitude, $f$ is the flapping frequency, and $\phi$ is the phase difference between the rotation and translation. In the present work, we choose $\alpha_{0}=-\frac{\pi}{2}$ and $\phi=0$, which corresponds to the symmetrical rotation [14]. The wing is assumed to be rigid and flights closely to the obstacle surface. The obstacle is represented by a circle, of which the center is at the middle of the stroke plane and the diameter is denoted as $d$. The distance between the center of the wing when it's vertical and the top of obstacle is defined as the $D$. We vary $d$ and $D$ to study the obstacle effect.

The flow is governed by the viscous incompressible Navier-

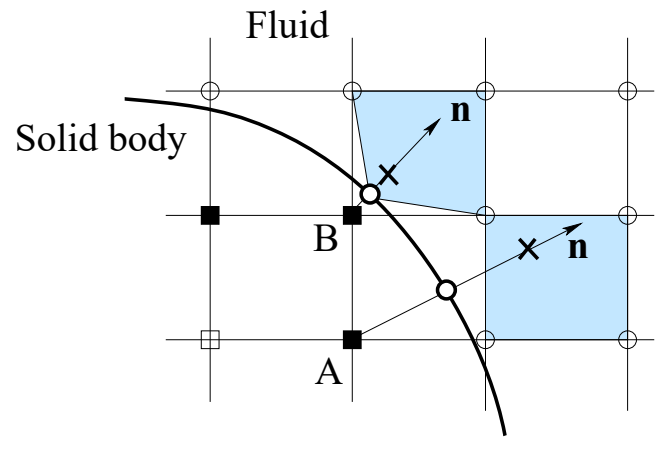

$\square$ Solid node $\quad \bigcirc$ Fluid node

- Ghost node (GN)

○ Body intercept (BI) $\times$ Image point (IP)

FIGURE 2. A 2D ILLUSTRATION OF THE SHARP-INTERFACE IMMERSED-BOUNDARY METHOD FOR THE FLUIDSOLID BOUNDARY. FLOW FIELD EXTRAPOLATION IS APPLIED AT THE GHOST NODES.

Stokes equation and the continuity equation,

$$
\begin{aligned}
\frac{\partial v_{i}}{\partial t}+\frac{\partial v_{j} v_{i}}{\partial x_{j}} & =-\frac{1}{\rho_{f}} \frac{\partial p}{\partial x_{i}}+v_{f} \frac{\partial^{2} v_{i}}{\partial x_{j}^{2}} \\
\frac{\partial v_{i}}{\partial x_{i}} & =0
\end{aligned}
$$

where $v_{i}$ is the velocity, $\rho_{f}$ and $v_{f}$ are the fluid density and viscosity, and $p$ is the pressure. No-slip and no-penetration conditions are specified at the flow-solid boundary. To parametrize the system, we define the non-dimensional groups including the normalized wing stroke, Reynolds number, normalized obstacle diameter and normalized distance, which are given by

$$
\frac{A}{c}, \quad \operatorname{Re}=\frac{\pi A f c}{v_{f}}, \quad \frac{d}{c}, \quad \frac{D}{c}
$$

The equations governing the system, e.g. Eqn. (3), are solved numerically in an implicitly coupled manner using an inhouse solver. Specifically, the incompressible flow is solved using a sharp-interface immersed-boundary method $[15,16]$ with a special treatment to suppress the pressure oscillations associated with the moving boundaries [17]. In this method, a single-block Cartesian grid is used to discretize the Navier-Stokes equation on a rectangular domain, and the ghost nodes and hybrid nodes are defined near the fluid-solid interface to facilitate the boundary treatment at the interface (Fig. 2). The infinitely thin membranous wing is augmented with an artificial thickness that is about three times of spacing of the Cartesian grid and is automatically reduced as the grid is refined. The wing section is discretized by a set of Lagrangian points initially distributed uniformly along the wing. 


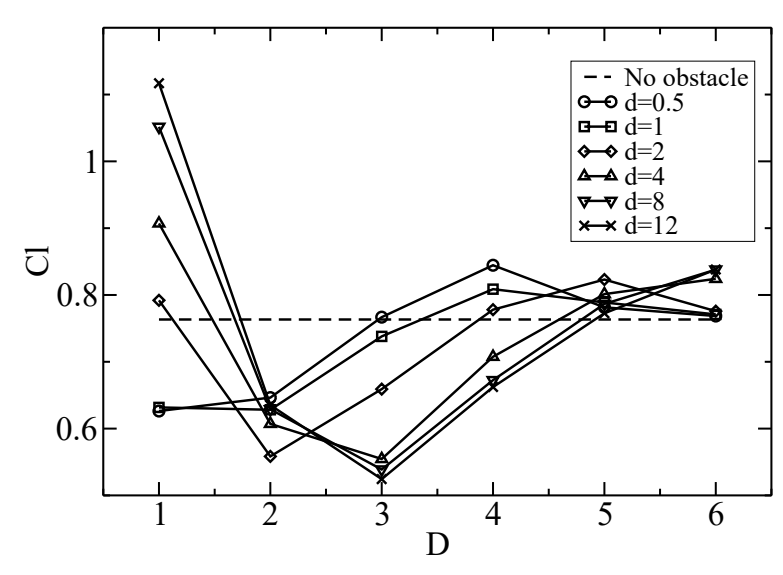

FIGURE 3. LIFT COEFFICIENT VS. DISTANCE.

\section{Simulation Setup}

In the present simulations, we choose the stroke distance $A / c=2.5$, Reynolds number $R e=200$, and rotational angle $\beta=\pi / 4$. The parameters describing the wing kinematics are selected based on previous work on insect flight $[13,18]$., representing the normal hovering flight in literature. The normalized obstacle diameter $d / c$ is chosen to vary among $0.5,1,2,4,8$ and 12. When the obstacle size becomes larger, the effect approaches the ground effect in previous studies. The normalized distance $D / c$ ranges from 1 to 6 with unit increment.

The computational domain has a size of $20 c \times 35 c$. We have done extensive tests to make sure that the domain is large enough to achieve satisfactory accuracy of the results. The entire domain consists of a nonuniform Cartesian grid of $320 \times 528$ points. The grid contains a horizontal band and a vertical band of width $3 c$ and $9 c$ in which the grid points are uniformly and densely distributed such that the grid spacings $\Delta x=\Delta y=0.02 c$. A total number of 100 nodes are used to discretize the wing. The time step size is $\Delta t=0.0025 T$ where $T=1 / f$ is the period of a flapping cycle. Fluid solver has been validated by comparing the computational results with literature. In addition, grid refinement has been performed to make sure that the simulation results are grid-independent.

\section{RESULTS AND DISCUSSION Large Obstacle Effect}

By changing the governing parameter $D(D$ and $d$ are normalized value in the following paper unless noted), lift and drag forces are calculated and nondimensionalized. The lift and drag coefficients are defined by Eqn. 5 and 6 respectively, which means the nondimensional vertical and horizontal forces.

$$
\begin{gathered}
C_{L}=\frac{2 F_{L}}{\left(\rho_{f} U^{2} c\right)}, \\
C_{D}=\frac{2 F_{D}}{\left(\rho_{f} U^{2} c\right)}
\end{gathered}
$$

where $F_{L}$ and $F_{D}$ represent the vertical and horizontal forces from the fiction and pressure acting on the wing.

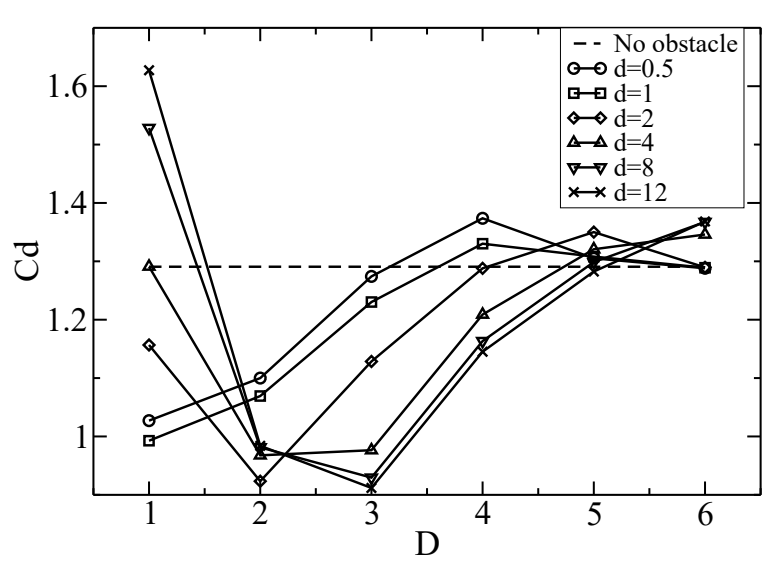

FIGURE 4. DRAG COEFFICIENT VS. DISTANCE.

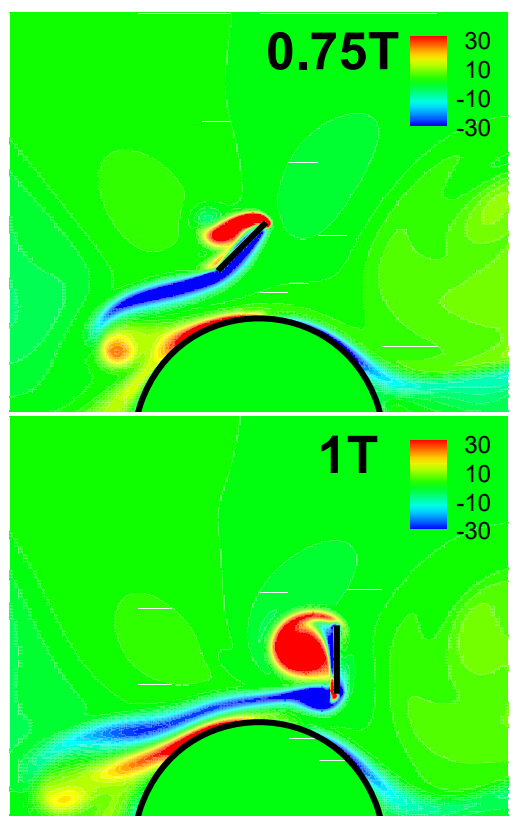

FIGURE 5. VORTICITY CONTOUR FOR $d=4$ AND $D=1$. THE CONTOUR LEVEL RANGES FROM $-30 U / c$ TO $30 U / c$

Fig. 3 and 4 shows how the lift and drag coefficients change with respect to $D$ for different sized obstacles. For $d>1$ the $C_{L}$ and $C_{D}$ change similarly as that under infinite ground effect. Take $d=4$ as example: when $D=1$, the LEV and TEV are generated during the translational and rotational motion and the stall delay occurs during the stroke, which contributes substantially to the high lift force. Because the wing gets so close to the obstacle $(D=1)$ and the obstacle size is large enough to encounter the shedding vortex from the wing. In Fig. 5 , the TEV is heavily stretched when it interacts with the obstacle and squeezed out the small gap. After that, the TEV shed quickly to the opposite direction. Such strong interaction between the TEV and obstacle creates strong vertical and horizontal effects, which leads to high $C_{L}$ and $C_{D}$ values in Fig. 3 and 4 at $D=1$.

As $D$ increases to 3 , the $C_{L}$ and $C_{D}$ reach the smallest value 


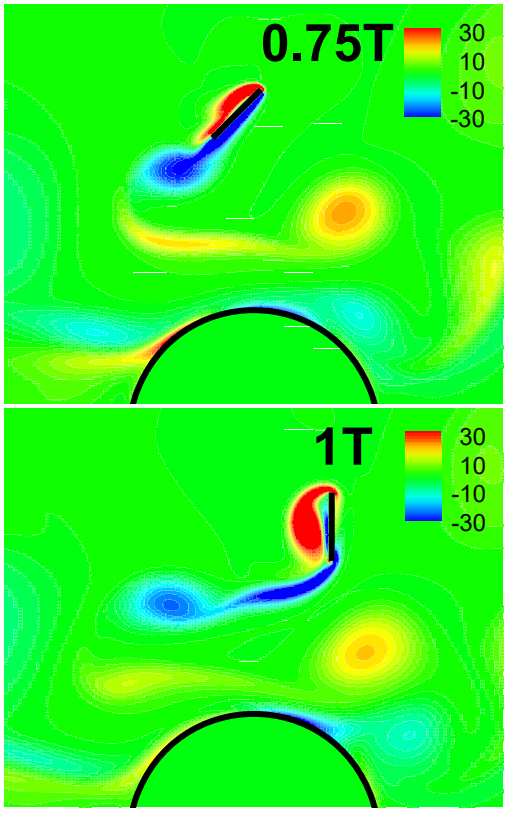

FIGURE 6. VORTICITY CONTOUR FOR $d=4$ AND $D=3$. THE CONTOUR LEVEL RANGES FROM $-30 U / c$ TO $30 U / c$

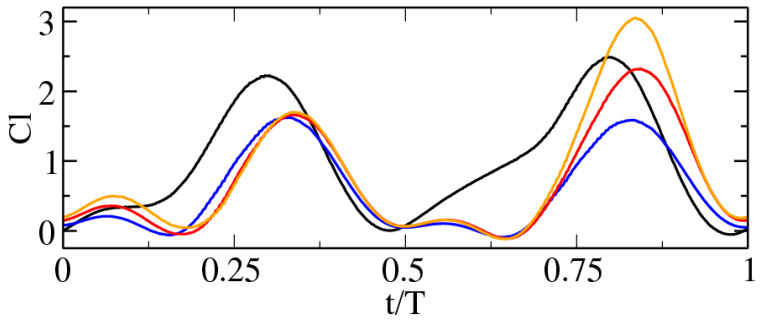

FIGURE 7. LIFT COEFFICIENT HISTORY IN ONE CYCLE: $d=4$ AND $D=1$ (BLACK), 3(BLUE), 4(RED) AND 6(ORANGE).

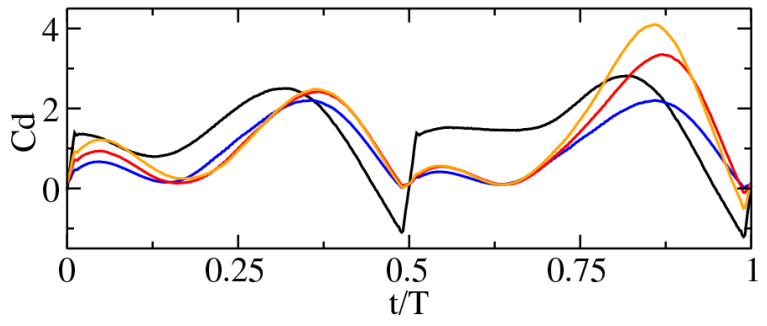

FIGURE 8. DRAG COEFFICIENT HISTORY IN ONE CYCLE: $d=$ 4 AND $D=1$ (BLACK), 3(BLUE), 4(RED) AND 6(ORANGE).

on the curves. The gap between the wing and obstacle increaes and the interaction between the wing and obstacle becomes less intensive. As shown in Fig. 6, the LEV becomes smaller compared that in Fig. 5, which indicates less energy the wing could get when both stall delay mechanism and wake capture effect are weakened. So it results in decreased lift force remarkably. The shedding TEV no longer directly interacts with obstacle and

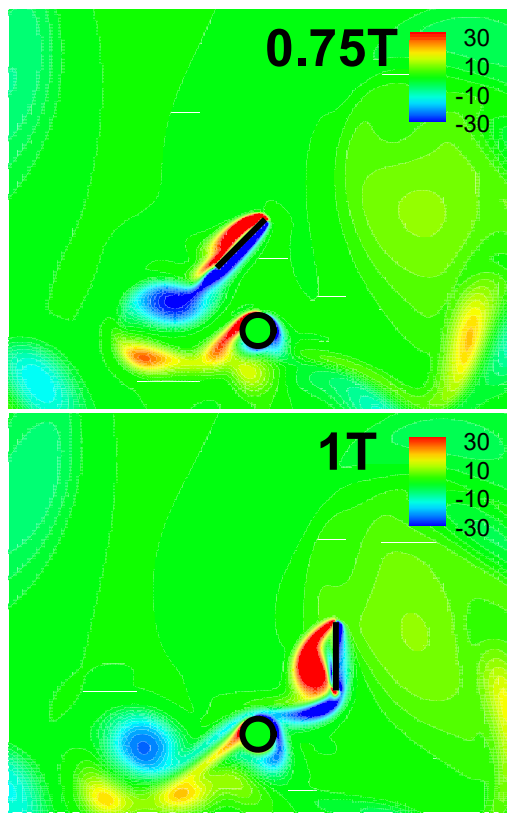

FIGURE 9. VORTICITY CONTOUR FOR $d=0.5$ AND $D=1$. THE CONTOUR LEVEL RANGES FROM $-30 U / c$ TO $30 U / c$

it merges with the previous shed vortex and dissipates gradually. Horizontal squeeze effect disappears so that drag force decreases. Complex vortex field leads to further horizontal and vertical force change. From these observation, the wing does not benefit from the interaction with the obstacle.

Fig. 7 and 8 shows the time history of lift and drag coefficients It shows that under the obstacle effect, at $D=1$, the lift reaches almost highest values during forward stroke and still keeps high value during backward stroke. For the forward stroke, the lift curves almost overlap each other for $D=3,4,6$. However, when the wing flaps backwards, because of different vortex structure and interaction with obstacle, lift turn out smallest at $D=3$ and largest at $D=6$. This explains why $C_{L}$ reaches valley in Fig. 3 and 4 at $D=3$. The drag history shows similar tendency as the lift history. Strong interaction during the backward flapping leads to higher drag force at $D=1$. Peak values for both lift and drag occur when the wing approach the cycle end at $D=6$, which means forces generated from wake capture may affected by obstacle effect.

\section{Small Obstacle Effect}

As shown in Fig 3 and Fig 4, new tendency can be observed for $d=0.5$ and 1 , which means that the obstacle size is smaller than or comparable with the flapping wings. Contrary with large obstacle effect, these two curves reach lowest value at $D=1$ and as the distance increases, both lift and drag increase. The values comes to peak when $D=4$, then converge to the value of free space flight, which is same as that of larger obstacles. Such observation means that the interaction between wing and small obstacle differs totally from that of large obstacle, which is analogous to infinite ground effect. Also the vortex field could be completely different such that smaller distance generates less 


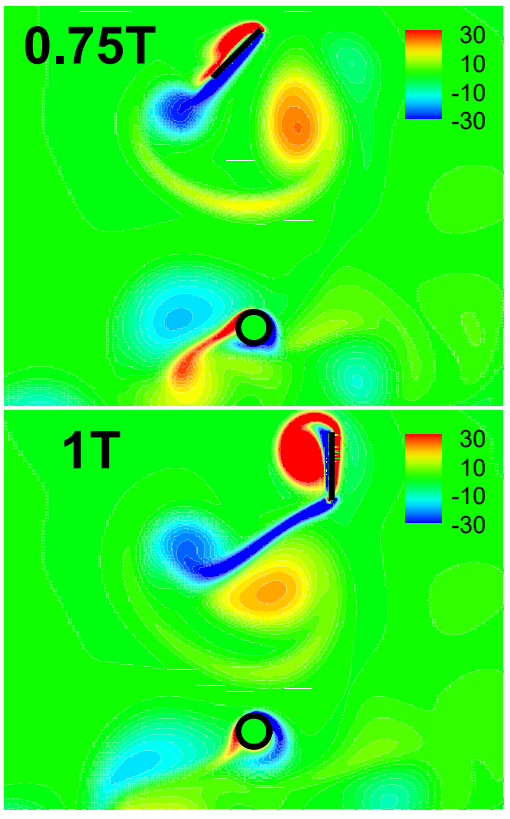

FIGURE 10. VORTICITY CONTOUR FOR $d=0.5$ AND $D=4$. THE CONTOUR LEVEL RANGES FROM $-30 U / c$ TO $30 U / c$

aerodynamic forces.

To analyze the obstacle effect, the vortex field is investigated for $d=0.5$ at $D=1$, which typically represents the nonconventional near surface hovering. Compare Fig. 9 and 10, the LEV at the end of stroke is much stronger at $D=4$ than at $D=1$, which explains the larger lift generation at $D=4$. For $d=4$, such comparison leads to opposite result. Fig. 9 shows different vortex interaction from that in Fig. 5. Because of the small size, the obstacle is not able to form a intensified vortex interaction region between the wing and its surface. The LEV becomes weaker as well as the TEV. The shedding vortex encapsule the obstacle and pass through it such that no elongation and squeeze-out occur, which may generate weaker horizontal forces. Also the shed vortex attach to the obstacle and dissipate with previous attached vortex. The obstacle disturbs the shedding vortex structure and its small size can not compensate the energy loss.

At $D=4$, the LEV evidently increases such that lift force consequently is enhanced by the stall delay mechanism. Because of the small size of the obstacle, the hovering flight somehow get close to the free space motion. TEV is fully developed, more elongated and shed into the area around the wing. From Fig. 3 and Fig. 1, the $C_{L}$ and $C_{D}$ are 0.84 and 1.37, which is comparable with the values of free space hovering.

Fig. 11 and 12 shows that during forward stroke at with $d=$ 0.5 , the lift and drag curves are close to each other. Compare the vortex field at $D=1$ and 4 when $\mathrm{t}=0.75 \mathrm{~T}$ (Fig 9 and 10), the LEV and TEV attached on the flapping share similar shape and strength. When the wing flap backward, force at further distance gradually becomes larger after $t=0.75 T$. Then the force reaches peak value at about $t=0.8 T$. At $t=1 T$, vortex structures show completely different status in Fig 9 and 10.

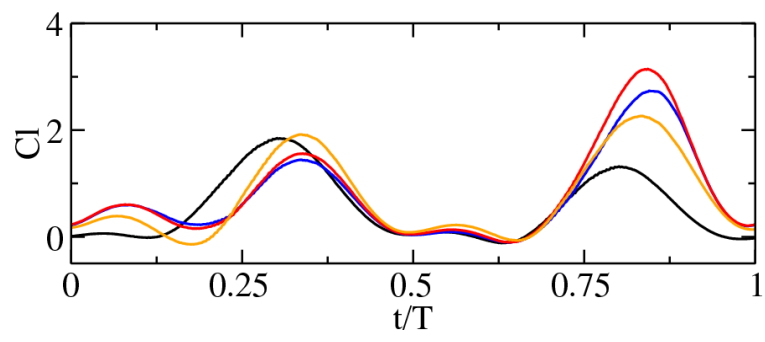

FIGURE 11. LIFT COEFFICIENT HISTORY IN ONE CYCLE: $d=$ 0.5 AND $D=1$ (BLACK), 3(BLUE), 4(RED) AND 6(ORANGE).

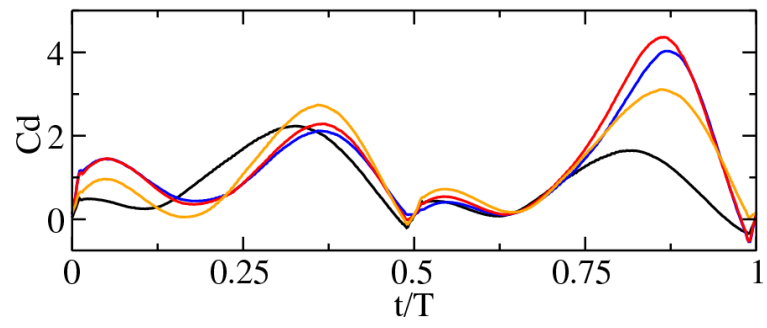

FIGURE 12. DRAG COEFFICIENT HISTORY IN ONE CYCLE: $d=0.5$ AND $D=1$ (BLACK), 3(BLUE), 4(RED) AND 6(ORANGE).

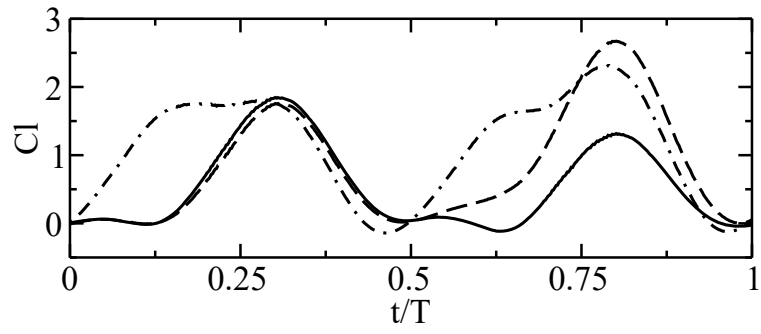

FIGURE 13. LIFT COEFFICIENT HISTORY IN ONE CYCLE: $D=$ 1 AND $d=0.5$ (SOLID), 2(DASH), AND 12(DASH DOT).

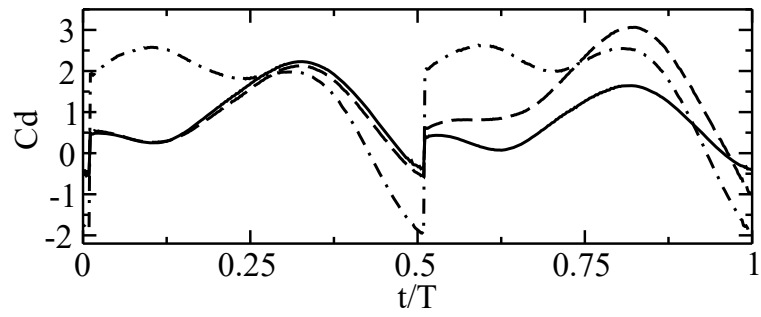

FIGURE 14. DRAG COEFFICIENT HISTORY IN ONE CYCLE: $D=1$ AND $d=0.5$ (SOLID), 2(DASH), AND 12(DASH DOT).

\section{Comparison Of Size Effect}

When the wing hovers away from the obstacle, the surface effect gradually decays. $C_{L}$ and $C_{D}$ curves converge to the values when no obstacle exists. As the wing approaches the obstacle, the effect becomes stronger. However, obstacle affects the aerodynamic performance differently due to their dimensions. When 
(A)

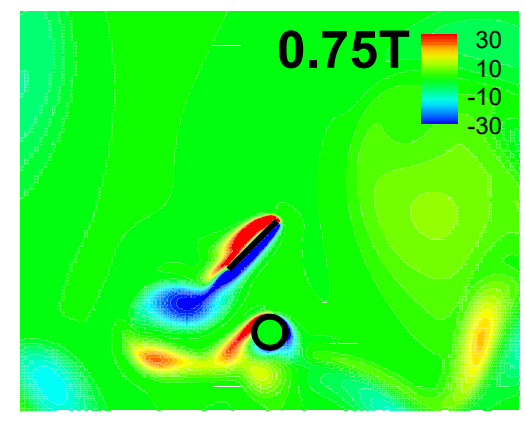

(B)

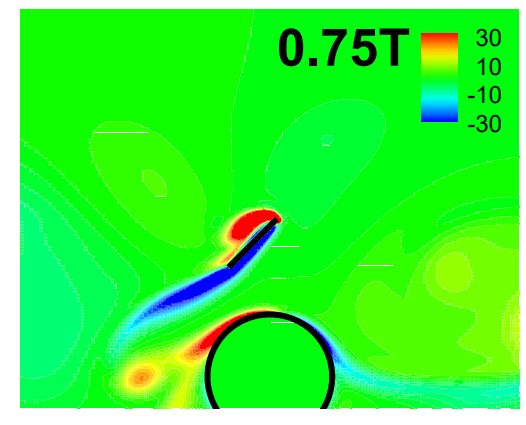

(C)

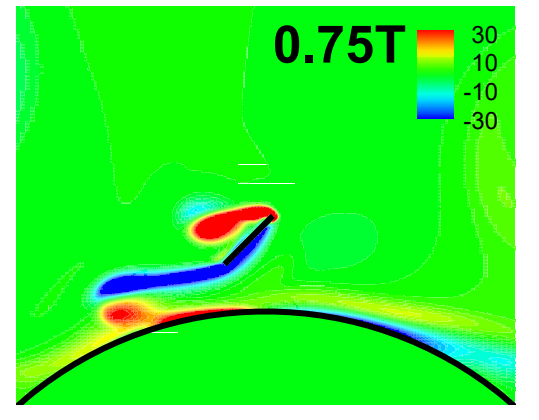

FIGURE 15. VORTICITY CONTOUR FOR $D=1$ : (A) $d=0.5$, (B) $d=2$ and (C) $d=12$. THE CONTOUR LEVEL RANGES FROM $-30 U / c$ TO $30 U / c$

$D=1$, the size effect differs most. Fig. 13 and 14 display the force histories in one cycle as the $D=1$. For $d=12$, which means the obstacle is large enough as ground, the lift and drag both are larger than the finite obstacles with $d=0.5$ and 2 during the forward stroke. During the backward stroke, the lift and drag of $d=0.5$ are evidently lower than the other two situations. By showing the vortex fields in Fig. 15, the shedding of LEV in (A) lags compared with (B) and (C). Also the shedding of TEV encounters the small obstacle, which may also cause the drag decrease.

\section{CONCLUSION}

In this paper, the fluid-structure interaction of a twodimensional hovering wing is numerically simulated in order to investigate the obstacle effect on the aerodynamic perfor- mance. The sharp immersed boundary method is used to solve 2D Navier-Stokes equations and simulation the hovering flight. Inset hovering flight is simplified and modeled by sinusoidal functions The obstacle diameter and distance effect is investigated under different combinations. During the hovering closely to the obstacle, the flow field becomes asymmetrical and vortex structure turns out complex. When obstacle size is larger than the wing, that is $d>=2$ the tendency of aerodynamic performance change with respect to distance show similarity when infinite ground effect exists. However, if the obstacle size is smaller or comparable with wing dimension $(d<=1)$, the aerodynamic change is opposite. Closer distance leads to smaller lift and drag generation, which is contrary to ground effect.

The results obtained in this study are helpful in understanding the mechanisms of finite obstacle effect when the insect hovers close to object and also provide information for MAV design.

\section{ACKNOWLEDGMENT}

This work is supported by the National Natural Science Foundation of China (Grant No. 11642022).

\section{REFERENCES}

[1] Ellington, C. P., 1999. “The novel aerodynamics of insect flight: applications to micro-air vehicles". Journal of Experimental Biology, 202(23), pp. 3439-3448.

[2] Pesavento, U., and Wang, Z. J., 2009. "Flapping wing flight can save aerodynamic power compared to steady flight". Physical review letters, 103(11), p. 118102.

[3] Lian, Y., Shyy, W., Viieru, D., and Zhang, B., 2003. "Membrane wing aerodynamics for micro air vehicles". Progress in Aerospace Sciences, 39(6), pp. 425-465.

[4] Kato, N., and Kamimura, S., 2008. Bio-mechanisms of swimming and flying: fluid dynamics, biomimetic robots, and sports science. Springer Science \& Business Media.

[5] Ellington, C. P., Van Den Berg, C., Willmott, A. P., and Thomas, A. L., 1996. "Leading-edge vortices in insect flight". Nature, 384(6610), p. 626.

[6] Birch, J. M., and Dickinson, M. H., 2003. "The influence of wing-wake interactions on the production of aerodynamic forces in flapping flight". Journal of Experimental Biology, 206(13), pp. 2257-2272.

[7] Dickinson, M. H., Lehmann, F.-O., and Sane, S. P., 1999. "Wing rotation and the aerodynamic basis of insect flight". Science, 284(5422), pp. 1954-1960.

[8] Wang, Z. J., 2005. "Dissecting insect flight". Annu. Rev. Fluid Mech., 37, pp. 183-210.

[9] Shyy, W., Trizila, P., Kang, C.-k., and Aono, H., 2009. "Can tip vortices enhance lift of a flapping wing?". AIAA journal, 47(2), pp. 289-293.

[10] Gao, T., and Lu, X.-Y., 2008. "Insect normal hovering flight in ground effect". Physics of Fluids, 20(8), p. 087101.

[11] Su, J.-Y., Tang, J.-H., Wang, C.-H., and Yang, J.-T., 2013. "A numerical investigation on the ground effect of a flapping-flying bird". Physics of Fluids, 25(9), p. 093101. 
[12] Wang, L., and Yeung, R. W., 2016. "Investigation of full and partial ground effects on a flapping foil hovering above a finite-sized platform". Physics of Fluids, 28(7), p. 071902.

[13] Eldredge, J. D., 2007. "Numerical simulation of the fluid dynamics of $2 \mathrm{~d}$ rigid body motion with the vortex particle method". J. Comput. Phys., 221, pp. 626-648.

[14] Wang, Z., Birch, J., and Dickinson, M., 2004. "Unsteady forces and flows in low Reynolds number hovering flight: two-dimensional computations vs robotic wing experiments". J. Exp. Biol., 207(3), pp. 449-460.

[15] Mittal, R., Dong, H., Bozkurttas, M., Najjar, F. M., Vargas, A., and vonLoebbeck, A., 2008. "A versatile sharp interface immersed boundary method for incompressible flows with complex boundaries". J. Comput. Phys., 227(10). 48254852.

[16] Luo, H., Mittal, R., Zheng, X., Bielamowicz, S. A., Walsh, R. J., and Hahn, J. K., 2008. "An immersed-boundary method for flow-structure interaction in biological systems with application to phonation". J. Comput. Phys., 227(22), pp. 9303-9332.

[17] Luo, H., Yin, B., Dai., H., and Doyle, J. F., 2010. "A 3D computational study of the flow-structure interaction in flapping flight". AIAA Paper 2010-556.

[18] Vanella, M., Fitzgerald, T., Preidikman, S., Balaras, E., and Balachandran, B., 2009. "Influence of flexibility on the aerodynamic performance of a hovering wing". J. Exp. Biol., 212(1), pp. 95-105. 